\title{
Influência da prática de exercícios físicos sobre a flexibilidade, força muscular manual e mobilidade funcional em idosos
}

\author{
Influence of physical exercise on the flexibility, hand muscle strength and functional \\ mobility in the elderly
}

\section{Resumo}

O processo de envelhecimento envolve alterações em todo o organismo humano, com declínio em quase todas as funções. A prática regular de exercício físico é uma estratégia atrativa e eficaz para retardar essas perdas. O objetivo deste estudo foi avaliar os resultados da prática de exercícios físicos, sobre a força muscular manual, flexibilidade e mobilidade funcional em idosos usuários de unidades de saúde da cidade de UberabaMG. Participaram 74 idosos (56 mulheres e 18 homens), pareados por sexo e idade, divididos em dois grupos com 37 indivíduos cada um. Foram avaliadas a força muscular manual, flexibilidade e mobilidade funcional. Os dados foram analisados utilizando o teste de Mann-Whitney $(\mathrm{p}<0,05)$. Houve diferença significativa entre os grupos com relação à flexibilidade. Evidencia-se a eficácia do exercício físico supervisionado nas unidades de saúde com relação à flexibilidade e indica-se a importância de modificar os treinos, implementando-se um protocolo mais definido com relação à força.

\section{Abstract}

The aging process involves whole bodily changes and decline in almost all functions. The regular practice of physical exercise is an attractive and effective strategy for slowing the losses. This study aimed to evaluate the results of physical exercise on the hand muscle strength, flexibility and functional mobility in elderly users of basic healthcare units in Uberaba, State of Minas Gerais, Brazil. Seventy four elderly participated (56 women and 18 men), matched by sex and age, divided in two groups with 37 individuals each. Hand muscle strength, functional flexibility and mobility were evaluated. Data were analyzed using the Mann-Whitney test $(\mathrm{p} \leq 0.05)$. There were significant differences for the two groups concerning flexibility. This study highlights the effectiveness of supervised exercise in the basic healthcare units with respect to flexibility and indicates the importance of modifying training, implementing a more defined protocol with respect to strength.
Palavras-chave: Idoso. Força Muscular. Flexibilidade. Exercício. Catalepsia.

Key words: Elderly. Muscle Strength. Flexibility. Exercise. Catalepsy.

\footnotetext{
Hospital das Clínicas da Faculdade de Medicina da Universidade de São Paulo. São Paulo, SP, Brasil.

2 Departamento de Fisioterapia Aplicada, Instituto de Ciências da Saúde. Universidade Federal do Triângulo Mineiro. Uberaba, MG, Brasil.
} 


\section{INTRODUÇÃO}

No Brasil, o ritmo de crescimento da população idosa tem sido sistemático e consistente. $\mathrm{O}$ envelhecimento da população brasileira é reflexo de uma taxa de fecundidade abaixo do nível de reposição populacional e do aumento da expectativa de vida, fazendo com que hoje o grupo de idosos ocupe espaço significativo na sociedade brasileira. ${ }^{1}$

O processo de envelhecimento envolve alterações em todos os sistemas do organismo humano, observando-se declínios significativos em quase todas as funções, nos diferentes componentes da capacidade funcional, em especial nas expressões da força muscular e na flexibilidade..$^{2,3}$

Com relação à força muscular, especialmente a força de preensão das mãos, Fleck \& $\mathrm{Kraemer}^{4}$ descreveram uma regressão de 3\% ao ano em homens e 5\% ao ano em mulheres, após estudo longitudinal com duração de quatro anos.

A flexibilidade pode ser definida como a máxima amplitude fisiológica passiva de determinado movimento articular. É considerada um dos componentes da aptidão e desempenho físico, sendo relevante para a execução de movimentos simples ou complexos, desempenho desportivo, manutenção da saúde e preservação da qualidade de vida. Não há definição de padrões ótimos de flexibilidade, bem como sua variação em função da idade, gênero, raça e padrão de atividade física regular. ${ }^{5}$ Sua perda não só reduz a quantidade e a natureza do movimento realizado por uma articulação, como pode ainda aumentar a probabilidade de lesão nessa articulação ou nos músculos envolvidos. ${ }^{6}$ É, sem dúvida, uma capacidade física muito importante nessa faixa etária, na medida em que é das principais responsáveis pela aptidão de realizar os movimentos diários com maior ou menor facilidade, como por exemplo, apertar os sapatos, pentear o cabelo, alcançar um armário, escovar os dentes, entre outras.

A elasticidade dos tendões, ligamentos e cápsulas articulares diminui com a idade devido à deficiência de colágeno, ${ }^{7}$ determinando que adultos percam algo como 8 a $10 \mathrm{~cm}$ de flexibilidade na região lombar e no quadril, quando medido por meio do teste sit and reach. ${ }^{8}$

A perda de força, associada à diminuição da flexibilidade em todas as articulações, afeta o equilíbrio, a postura e o desempenho funcional, aumenta o risco de quedas e problemas respiratórios, diminui a velocidade da marcha e dificulta as atividades da rotina diária. ${ }^{2} \mathrm{O}$ movimento de passar de sentado para em pé é uma das atividades mais executadas na vida diária de um indivíduo. É um movimento complexo cuja realização é pré-requisito para garantir uma postura em pé e essencial para o início da marcha. ${ }^{10}$

Consequentemente, a manutenção ou ganho de flexibilidade e força muscular é uma meta importante no controle da saúde de idosos. Dentre as alternativas para minimizar as perdas de força muscular, está a prática de exercício físico regular que, segundo Faria et al., ${ }^{11}$ possibilita a melhora ou manutenção da autonomia do idoso e sua inserção social. Ainda, Rauchbach ${ }^{12}$ encontrou relação entre essa prática e a melhora da amplitude de movimento nas articulações dos membros superiores e inferiores.

Neste sentido, os programas de exercícios físicos voltados para o desenvolvimento da força muscular e flexibilidade têm sido recomendados como meio de atenuar ou reverter os efeitos negativos relacionados ao envelhecimento e/ou fatores a ele associados, sobre esses componentes da capacidade funcional, ${ }^{13}$ diminuindo os efeitos do ciclo imobilidade-quedas/dor/medoimobilidade. $^{14}$ Podem ser utilizados como estratégia preventiva primária, para manter e melhorar o estado de saúde em qualquer idade, com efeitos benéficos diretos e indiretos na prevenção e retardamento das perdas funcionais, reduzindo o risco de enfermidades e transtornos frequentes do envelhecimento. ${ }^{15-17}$

Na rede de atenção básica (unidades básicas de saúde e estratégias de saúde da família) da cidade de Uberaba-MG, existe a prática de programas coletivos que acontecem duas vezes por semana. Realizam-se atividades de educação em saúde, 
dinâmicas de relaxamento e interação social pelos diferentes profissionais e estudantes universitários envolvidos e exercícios físicos orientados. Estas são realizadas pelos fisioterapeutas e estudantes de Fisioterapia, sob orientação de um professor responsável, com o objetivo de melhorar a qualidade de vida, aumentar a reserva funcional, prevenir e minimizar as limitações, evitar manifestações das doenças, controlar as já existentes, promover mudanças de hábitos e prevenir traumas e acidentes.

Os exercícios realizados nos grupos são orientados, mas não seguem um protocolo definido, variando quanto à intensidade e tempo para cada um. Em todos os grupos são realizados alongamentos globais, fortalecimento com e sem resistência, treino de equilíbrio estático e dinâmico e exercícios de coordenação motora.

Por se tratar de uma atividade em expansão no sistema público de saúde, há necessidade de se avaliar os resultados da prática de exercícios físicos sobre a força muscular manual, flexibilidade e mobilidade funcional em idosos, para que seja possível oferecer subsídios ao direcionamento de programas de promoção, comportamento mais ativo dessa população e ampliação da abrangência destas atividades na rede pública, com impactos positivos na saúde.

Nesse contexto, o objetivo deste estudo foi avaliar os resultados da prática de exercícios físicos sobre a força muscular manual, flexibilidade e mobilidade funcional em idosos usuários de unidades de saúde da cidade de Uberaba-MG.

\section{METODOLOGIA}

\section{Identificação do tipo de estudo e população}

Estudo descritivo, transversal, de caráter exploratório e metodologia quantitativa. A amostra de conveniência foi composta por idosos de ambos os sexos, com idade igual ou acima de 60 anos, participantes do Programa PET de Saúde da Universidade Federal do Triângulo Mineiro (UFTM), na cidade de UberabaMG. Participaram idosos sedentários (que não praticam atividades físicas além das necessidades diárias e que se utilizam dos serviços da rede de atenção básica de saúde), e idosos ativos fisicamente (que praticam atividades físicas com frequência mínima de um dia por semana e que participam dos programas coletivos oferecidos pela rede de atenção básica onde se desenvolve o programa PET Saúde da UFTM.

Foram excluídos idosos com incapacidade de compreensão para responderem aos questionários ou incapacidades físicas que os impedissem de realizar os parâmetros utilizados na avaliação física e aqueles que não assinaram o Termo de Consentimento Livre e Esclarecido. A coleta de dados foi realizada no período de abril a setembro de 2011.

\section{Procedimento para coleta de dados}

A avaliação foi realizada por meio de entrevistas e avaliação física individual. Para os idosos do Grupo de Praticantes (GP), estas foram realizadas no próprio local e horário onde o idoso realiza a atividade física. Os idosos do Grupo de Não-Praticantes (GNP) foram avaliados na própria unidade de saúde em uma sala reservada, quando iam até a mesma em busca de algum atendimento ou em suas próprias casas, em horário previamente agendado, conforme maior comodidade destes, por um fisioterapeuta previamente treinado. Para manutenção do sigilo, os questionários foram numerados.

Instrumentos para a coleta de dados

A força muscular manual foi aferida por meio de um dinamômetro hidráulico de mão (preensão palmar), de acordo com a técnica proposta por Bechtol. ${ }^{18}$ Os indivíduos permaneceram sentados, com o ombro do membro superior a ser avaliado em posição neutra, cotovelo a 90 graus e punho neutro. Foram aferidas três medidas e considerado para o estudo o maior valor atingido.

A flexibilidade foi avaliada por meio do teste de sentar e alcançar com a utilização do 
Banco de Wells ${ }^{19}$ e teste do Alcance Funcional Anterior. ${ }^{20}$ A avaliação por meio do Banco de Wells foi realizada com o indivíduo sentado no chão, joelhos estendidos e pés completamente apoiados na parte anterior deste. Solicitouse que o mesmo levantasse os braços com as mãos sobrepostas, levando ambas para frente e empurrando o marcador para o mais distante possível na régua, sem impulsos ou insistência. $\mathrm{O}$ teste foi repetido por três vezes, sendo utilizada a maior medida.

No teste do Alcance Funcional Anterior, o indivíduo posicionou-se lateralmente a uma fita métrica colocada na parede à altura do ombro e flexionou o tronco o máximo que conseguiu, sem dar um passo. Foi utilizada a medida do deslocamento anterior sobre a fita métrica, em centímetros, tendo como parâmetro o terceiro metacarpo do indivíduo.

A mobilidade funcional foi avaliada por meio do Timed up and Go (TUG), ${ }^{21}$ que consiste no indivíduo levantar-se de uma cadeira, sem ajuda dos braços, andar a uma distância de três metros, dar a volta e retornar. No início do teste, o mesmo estava com o dorso apoiado no encosto da cadeira e, ao final, encostou novamente. $\mathrm{O}$ mesmo recebeu a instrução "vá" para realizar o teste e o tempo foi cronometrado a partir da vOz de comando até o momento em que ele apoiou novamente o dorso no encosto da cadeira. O TUG foi realizado uma vez para familiarização e uma segunda para tomada do tempo.
Análise dos dados

As diferenças entre os grupos foram analisadas por meio do teste de Mann-Whitney. O nível de significância adotado foi de $\mathrm{p}<0,05$.

\section{Aspectos legais da pesquisa}

O projeto foi aprovado pelo Comitê de Ética em Pesquisa da Universidade Federal do Triângulo Mineiro, sob protocolo $\mathrm{n}^{\mathrm{o}}$ 1.795. Os indivíduos foram informados sobre os objetivos e a metodologia do estudo e assinaram o Termo de Consentimento Livre e Esclarecido, conforme Resolução no 196, de 10 de outubro de 1996 do Conselho Nacional de Saúde, que regulamenta pesquisas que envolvem seres humanos.

\section{RESULTADOS}

Foram avaliados 74 idosos (sendo 56 mulheres e 18 homens), pareados por sexo e idade, divididos em dois grupos com 37 indivíduos cada, sendo o GP composto por 28 mulheres e nove homens com idades entre 60 e 83 anos (média $=69,8$ e $\mathrm{dp}=6,4)$, e GNP composto também por 28 mulheres e nove homens com idades entre $60 \mathrm{e}$ 87 anos (média $=69,9$ e dp=6,7).

Os valores das médias e desvio-padrão da força muscular manual, flexibilidade, alcance funcional e TUG, assim como os valores de "p" para as diferenças entre GP e GNP, podem ser observados na tabela 1.

Tabela 1 - Valores das médias de força muscular manual, flexibilidade, alcance funcional, TUG e valores de "p" para as diferenças entre os grupos GP e GNP. Uberaba, MG, 2011.

\begin{tabular}{lccc}
\hline & GP & GNP & P \\
& Média (DP) & Média (DP) & \\
\hline Idade (anos) & $69,78(6,4)$ & $69,95(6,7)$ & \\
Força muscular (kgf/cm) & $27,15(6,7)$ & $24,8(9,0)$ & 0,2984 \\
Flexibilidade (cm) & $23,26(10,6)$ & $15,99(9,3)$ & $0,0018^{*}$ \\
Alcance (cm) & $33,46(8,4)$ & $25,62(10,0)$ & $0,0008^{*}$ \\
TUG (seg) & $9,03(3,4)$ & $9,68(4,4)$ & 0,3844 \\
\hline
\end{tabular}

${ }^{1}$ Teste de Mann-Whitney $* \mathrm{p}<0,05$. 
Tomando como referência Shephard, ${ }^{22}$ que refere que a força de preensão manual aos 55 anos é de $34 \mathrm{Kgf}$, e que aos 75 anos cai para $22 \mathrm{Kgf}$, e que as medidas da força de preensão manual de idosos guardam relação diretamente proporcional ao quadro de força muscular geral, podemos considerar que, apesar de a média de idade dos idosos do presente estudo ter sido pouco inferior aos 75 anos usados como referência por esse autor, a média da força muscular nos dois grupos foi superior, indicando boa condição deste componente da capacidade funcional. No entanto, apesar de o GP apresentar média do valor de força de preensão manual maior que GNP, não foi encontrada diferença estatística significativa para os grupos.

No que se refere à flexibilidade, o GNP foi classificado entre abaixo da média e ruim; e o GP, entre média e abaixo da média, segundo Wells \& Dillon, ${ }^{19}$ indicando melhores condições para este último.

Quanto ao deslocamento anterior, Duncan et al. ${ }^{20}$ referem que aqueles menores que $15 \mathrm{~cm}$ indicam fragilidade e risco de quedas. Os idosos do presente estudo apresentaram médias maiores que este valor, indicando baixa propensão a quedas. Diferenças significativas entre os grupos foram encontradas para a avaliação por meio desses testes, indicando melhores condições para o GP.

Com relação à mobilidade funcional, segundo Nordin et al.,23 uma pontuação acima de 30 segundos significa alto risco de quedas; entre 20 e 30 segundos, moderado risco; e abaixo de 20 segundos, baixo risco de quedas. Nos idosos deste estudo, as médias dos valores para os dois grupos foram muito parecidas, classificando-os em baixo risco para quedas, não havendo diferença estatisticamente significativa entre os grupos.

\section{DISCUSSÃO}

Os resultados da força de preensão manual encontrados neste estudo foram semelhantes ao de Rebelatto, ${ }^{7}$ que analisou 32 idosas (de 60 a 80 anos) que praticaram exercícios físicos orientados durante dois anos, não encontrando resultados estatisticamente significativos para a diferença de força muscular manual destas.

Considerando-se, no entanto, a importância da força no processo do envelhecimento, seja nos membros inferiores presentes na marcha ou nos membros superiores para a realização das atividades vitais do dia a dia, como abrir uma lata com tampa de rosca, e perdas funcionais que ocorrem devido à diminuição da mesma, ${ }^{24,25}$ a simples manutenção desta força é sinal de que os exercícios realizados foram eficazes. A partir dos 65-70 anos, a perda de força torna-se mais grave e é responsável pelos consideráveis déficits motores observados em indivíduos nessa faixa etária.

Harries \& Bassey ${ }^{26}$ referem um declínio de $15 \%$ da força máxima individual entre a sexta e a sétima décadas de vida e de $30 \%$ a cada década, após este período. Ainda, a redução da força muscular pode variar para seus diferentes tipos de expressões, sobretudo para a potência. Algumas evidências têm demonstrado que o declínio da potência muscular pode ocorrer de maneira precoce (a partir da quinta década de vida) e com maior taxa (3,5\% ao ano) quando comparada à força muscular máxima. ${ }^{27}$

Faria et al. ${ }^{11}$ relatam que, entre as alternativas para minimizar as perdas de força muscular, está a prática de exercício físico regular, que possibilita a melhora ou manutenção da autonomia do idoso e sua inserção social. Desta maneira, o fato de não se encontrar diferença significativa entre os grupos avaliados no presente estudo indica a necessidade de se incrementar a prática de exercícios que visem ao fortalecimento muscular global nas atividades coletivas oferecidas a esses idosos.

Com relação à flexibilidade, Holland et al. ${ }^{28}$ referem que é possível observar um declínio de $20-50 \%$ desta entre os 30 e 70 anos. Desta maneira, a diferença significativa entre os grupos com relação a essa variável, neste estudo, confirma a importância da realização de exercícios físicos para sua manutenção. Os exercícios físicos impedem o declínio gradual das aptidões físicas, que podem alterar os hábitos de vida e rotinas diárias dos idosos, levando-os a realizar atividades e formas de ocupação pouco ativas. 
$\mathrm{Na}$ mesma direção, Feland et al., ${ }^{9}$ em estudo realizado com idosos, concluíram que alongamentos de 15 e 30 segundos repetidos quatro vezes em cada sessão, cinco vezes por semana, por seis semanas, aumentaram significativamente a amplitude de movimento em relação ao grupo-controle. Rauchbach ${ }^{12}$ encontrou relação entre a realização de exercícios físicos e melhora da amplitude de movimento nas articulações dos membros superiores e inferiores. A flexibilidade da coluna lombar, medida durante a flexão anterior de tronco, mostrou um aumento médio de $5 \mathrm{~cm}$ em 88,1\% dos participantes após o treinamento, demonstrando que a atividade física constante melhora a flexibilidade.

Apesar de o GNP ter sido classificado entre abaixo da média e ruim no teste de sentar e alcançar, e o GP entre média e abaixo da média, segundo Wells \& Dillon, ${ }^{19}$ indicando melhores condições para este último, os resultados indicam que os exercícios praticados nas atividades coletivas devem enfatizar ganhos de flexibilidade para que se alcancem melhores padrões com relação a esse parâmetro.

Com relação à mobilidade funcional, Terena \& Taricco ${ }^{29}$ mostraram a diferença entre o tempo total para levantar entre dois grupos (um de 25 a 35 anos e outro de 60 a 65 anos), executando o movimento de levantar de uma cadeira sem apoio. No movimento realizado com os olhos abertos, houve diferença entre a velocidade que o grupo de sujeitos de 25-35 anos levanta, em relação ao grupo de sujeitos de 60-65 anos, evidenciando que os movimentos ficam mais lentos com o passar dos anos.

Nos idosos deste estudo, as médias dos valores para os dois grupos foram muito parecidas, classificando-os em baixo risco para quedas, não havendo diferença estatisticamente significativa entre estes. Este resultado evidencia a necessidade de as atividades coletivas utilizarem exercícios que incrementem os ganhos de mobilidade, considerando-se a importância desta. O movimento de passar de sentado para em pé é uma das atividades mais executadas na vida diária de um indivíduo, e é um movimento complexo cuja realização é pré-requisito para garantir uma postura em pé e essencial para o início da marcha. ${ }^{10}$ Requer a habilidade de controlar o corpo durante o deslocamento do centro de gravidade de uma base de apoio ampla (cadeira) para uma base de apoio menor (em pé), retomando o equilíbrio assim que se assume a posição ereta. ${ }^{30}$

Assim, podemos observar que a maioria das perdas funcionais se acentua com a idade devido à insuficiente atividade do sistema neuromuscular, ao desuso e à diminuição do condicionamento físico, determinando complicações e condições debilitantes, inanição, desnutrição, ansiedade, depressão, insônia, etc. Estas, por sua vez, conduzem à imobilidade, desuso, debilidade muscular e enfermidades, estabelecendo um círculo vicioso clássico em Geriatria?

Desta maneira, como aumento da longevidade, parece clara a necessidade de maior atenção à variedade de estados possíveis das condições de saúde, sendo prioritária a intervenção preventiva numa perspectiva de melhorar a qualidade de vida. Essa intervenção evidencia a necessidade de se criar novas respostas por parte do Estado, da sociedade civil, das instituições e sobretudo dos profissionais de saúde, no desenvolvimento de ações que impeçam a influência das variáveis que determinam as más condições de saúde do indivíduo idoso, caracterizando melhor qualidade de vida. ${ }^{31}$ Dentre essas ações, encontrase a prática de exercícios físicos, uma vez que seus benefícios não se restringem somente ao campo físico-funcional e mental dos indivíduos, melhorando também o desempenho funcional, social, mantendo e promovendo a independência e a autonomia daqueles que envelhecem. ${ }^{32}$

\section{CONCLUSÃO}

Os resultados do presente estudo indicaram a importância de aprimorar a prática coletiva de exercícios físicos realizados nos serviços de atenção básica de saúde, com maior ênfase para força muscular, flexibilidade e mobilidade. Por se tratar de uma atividade em expansão no sistema público de saúde, espera-se que esta pesquisa contribua para o planejamento de outros estudos, 
com maior número de sujeitos e comparando a aplicação de diferentes protocolos. Os resultados devem disseminar a utilização desta prática de forma otimizada dentro das ações de promoção e prevenção de saúde, garantindo maiores ganhos, minimizando e retardando os efeitos do envelhecimento, e preservando a independência e saúde dessa população.

\section{REFERÊNCIAS}

1. Instituto Brasileiro de Geografia e Estatística. Síntese de indicadores sociais: uma análise das condições de vida da população brasileira 2009. [acesso em 12 set. 2011]. Disponível em: http://www.ibge.gov.br/home/estatistica/ populacao/condicaodevida/indicadoresminimos/ sinteseindicsociais2009/default.shtm

2. Mazzeo RS, Cavanag P, Evans WJ, Fiatarone MA, Hagberg J, McAuley E, et al. Exercício e atividade física para pessoas idosas. Rev Bras Ativ Fís Saúde 1998;3(1):48-78.

3. Gonçalves R, Gurjão ALD, Gobbi S. Efeitos de oito semanas do treinamento de força na flexibilidade de idosos. Rev Bras Cineantropom Desempenho Hum 2007;9(2):145-53.

4. Fleck SJ, Kraemer WJ. Fundamentos do treinamento de força muscular. Porto Alegre: Artmed; 1999.

5. Araújo CGS. Correlação entre diferentes métodos lineares adimensionais de avaliação da mobilidade articular. Rev Bras Ciên e Mov 2000;8(2):25-32.

6. Spirduso WW. Physical dimensions of aging. Champaign, IL: Human Kinetics; 1995. 432 p.

7. Rebelatto JR, Calvo JI, Orejuela JR, Portillo JC. Influência de um programa de atividade física de longa duração sobre a força muscular manual e a flexibilidade corporal de mulheres idosas. Rev Bras Fisioter 2006;10(1):127-32.

8. Shephard RJ. Aging and exercise. Encyclopedia of Sports Medicine and Science. Internet Society for Sport Science. 1998 [acesso em 12 set. 2011]. Disponível em: http://sportsci.org/

9. Feland JB, Myrer JW, Schulthies SS, Fellingham GW, Measom GW. The effect of duration of stretching of the hamstring muscle group for increasing range of motion in people aged 65 years or older. Phys Ther 2001;81(5):1110-7.

10. Shumway-Cook A, Woollacott MH. Motor control: theory and practical applications. Baltimore: Williams \& Wilkins; 1995.

11. Faria JC, Machala CC, Dias RC, Dias JMD. Importância do treinamento de força na reabilitação da função muscular, equilíbrio e mobilidade de idosos. Acta Fisiatr 2003;10(3):133-7.
12. Rauchbach R. A atividade física para terceira idade, analisada e adaptada. Curitiba: Lovise; 1990.

13. American College of Sports Medicine Position Stand. The recommended quantity and quality of exercise for developing and maintaining cardiorespiratory and muscular fitness, and flexibility in healthy adults. Med Sci Sports Exerc 1998;30(6):975-91.

14. Daley MJ, Spinks WL. Exercise, mobility and aging. Sports Med. 2000;29(1):1-12.

15. de Jong N, Chin A Paw MJ, de Groot LC, de Graaf C, Kok FJ, van Staveren WA. Functional biochemical and nutrient indices in frail erderly people are partly affected by dietary supplements but not by exercise. J Nutr. 1999;129:2028-36.

16. Lewis RD, Modlesky CM. Nutrition, physical activity, and bone health in women. Int J Sport Nutr 1998;8(3):250-84.

17. Polidori MC, Mecocci P, Cherubini A, Senin U. Physical activity and oxidative stress during aging. Int J Sports Med 2000;21(3):154-7.

18. Bechtol CO. Grip test; the use of a dynamometer wish adjustable handle spacings. J Bone Joint Surg Am 1954;36-A(4):820-4.

19. Wells KF, Dillon EK. The sit and reach: a test of back and leg flexibility. Res Q Exerc Sport 1952;23:115-8.

20. Duncan PW, Weiner DK, Chandler J, Studenski S. Functional reach: a new clinical measure of balance. J Gerontol 1990;45(6):M192-7.

21. Podsiadlo D, Richardson S. The timed "Up \& Go": a test of basic functional mobility for frail elderly persons. J Am Geriatr Soc 1991;39(2):142-8.

22. Shephard RJ. Envelhecimento, atividade física e saúde. São Paulo: Phorte; 2003.

23. Nordin E, Rosendahl E, Lundin-Olsson L. Timed "Up \& Go" test: reliability in older people dependent in activities of daily living--focus on cognitive state. Phys Ther 2006 May;86(5):646-55.

24. Kauffman TL. Manual de Reabilitação Geriátrica. Rio de Janeiro: Guanabara Koogan; 2001.

25. Bassey EJ. Physical capabilities, exercise and aging. Rev Clin Gerontol. 1997;7(4):289-97. 
26. Harries UJ, Bassey EJ. Torque-velocity relationships for the knee extensors in women in their 3rd and 7th decades. Eur J Appl Physiol Occup Physiol 1990;60(3):187-90.

27. Izquierdo M, Ibañez J, Gorostiaga E, Garrues M, Zúñiga A, Antón A et al. Maximal strength and power characteristics in isometric and dynamic actions of the upper and lower extremities in middle-aged and older men. Acta Physiol Scand 1999;167(1):57-68.

28. Holland GJ, Tanaka K, Shigematsu R, Nakagaichi M. Flexibility and physical functions of older adults: a review. J Aging Phys Act 2002;10(2):169-206.
29. Terena SML, Taricco MA. Comparação das estratégias musculares entre dois grupos etários diferentes no movimento de passar de sentado para em pé. Acta Fisiatr 2009;16(3):105-9.

30. Janssen WG, Bussmann HB, Stam HJ. Determinants of the sit-to-stand movement: a review. Phys Ther 2002;82(9):866-79.

31. Ministério da Saúde (Brasil). Diretrizes do NASF: Núcleo de Apoio a Saúde da Família. Série A. Normas e Manuais Técnicos. Caderno de Atenção Básica n. 27. Brasília: Ministério da Saúde, 2010. 152 p.

32. Zaitune MPA, Barros MBA, César CLG, Carandina L, Goldbaum M. Fatores associados ao sedentarismo no lazer em idosos, Campinas, São Paulo, Brasil. Cad Saúde Pública 2007;23(6):1329-38. 